Zbl Arbeitsmed 2016 $66: 115$

DOI 10.1007/s40664-016-0091-y

Online publiziert: 5. Februar 2016

๑) Springer-Verlag Berlin Heidelberg 2016

\title{
D. Nowak
}

Institut und Poliklinik für Arbeits-, Sozial- und Umweltmedizin, WHO Collaborating Centre

for Occupational Health, Klinikum der Universität München, München, Deutschland

\section{Prof. Dr. med. Alfred Manz}

\subsubsection{1-31.12.2015}

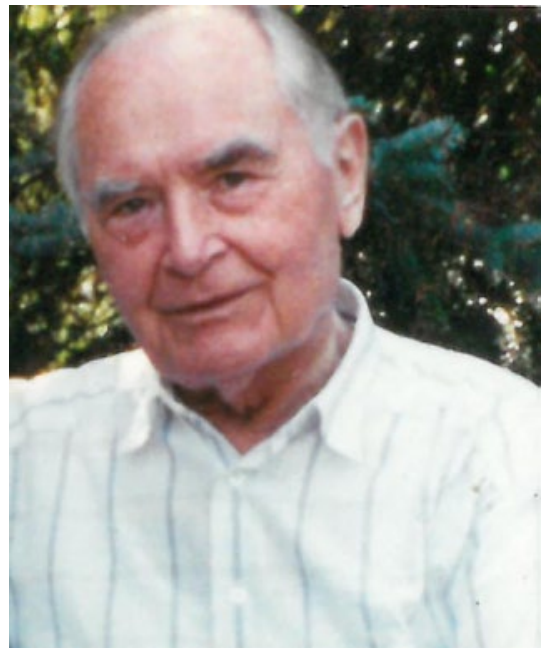

Alfred Manz

Der Hamburger Arbeitsmediziner Prof. Dr. Alfred Manz starb am 31.12.2015 im Alter von 94 Jahren.

Studenten der Medizin begeisterte er in den frühen 1980er Jahren in extracurriculären Seminaren für das „trockene“ Fach Arbeitsmedizin mit Betriebsbesichtigungen in großen Hamburger Betrieben. Wir waren fasziniert, wie er geradezu beiläufig hier eine Asbestplatte hervorzog, dort ein Benzol-Gebinde entdeckte - er machte Arbeitsmedizin für uns erlebbar.

Stolze 35 Jahre lang arbeitete Prof. Manz als Betriebsarzt bei den Hamburger Gaswerken. Zusammen mit dem Epidemiologen Prof. Dr. Jürgen Berger, Hamburg, legte er die wissenschaftliche Grundlage für die Berufskrankheit 4110 (Bösartige Neubildungen der Atemwege durch Kokereirohgase). Dieser Forschungsschwerpunkt fand seine Weiterentwicklung über die Berufskrankheiten 4113, 4114 und aktuelle Be- ratungen zum Larynx- und Lungenkarzinom bis in die heutige Zeit.

Weltweite Aufmerksamkeit erhielt Manz jedoch mit seinen Arbeiten über die „Boehringer-Kohorte“. Hierbei handelt es sich um Arbeiter aus der Herbizidproduktion mit beruflicher Exposition gegenüber Dibenzodioxinen und Dibenzofuranen. Seine erste wissenschaftliche Arbeit hierüber erschien 1991 im Lancet, seine letzte 2012 in Occupational Environmental Medicine. Der Senat der Freien und Hansestadt Hamburg machte ihn zum Leiter der Beratungsstelle für Chemiefacharbeiter in Hamburg, wo er sich bis zum Ende seiner Tage für erkrankte ehemalige BoehringerMitarbeiter einsetzte.

Nie werde ich vergessen, wie er mich ich war damals frischer Stationsarzt in der Lungenklinik Großhansdorf bei Hamburg, und es war wohl erstmals 1985 oder 1986 - immer wieder auf der Krankenstation besuchte, wenn bei einem der von ihm arbeitsmedizinisch betreuten Patienten mit auffälligem Lungenbefund eine Abklärung erforderlich war. Er drängte mich geradezu, eine ausführliche Arbeitsanamnese zu erheben und gab keine Ruhe, wenn die Themen "Asbest", „Kokereirohgase“ oder auch „Dioxin“ und „Benzol“ nicht komplett und quantitativ abgearbeitet waren. Auch andere Stationsärzte in anderen Kliniken machten diese Erfahrungen.

Prof. Manz widmete sich früh Fragen der Synkanzerogenese, der Risikoverdoppelung, der häuslichen Verschleppung beruflicher Kanzerogene - also den auch heute noch wichtigen und offenen Fragen zum Thema „Berufskrebs“.

Konflikte mit der „etablierten“ arbeitsmedizinischen Lehrmeinung blieben nicht aus. Manchmal rang der Kämpfer für Patientenrechte Manz mit dem Wissenschaftler Manz. Wir damaligen Studierenden und späteren Stationsärzte werden seine Worte nicht vergessen: „Wer soll sich denn um die Frage der beruflichen Verursachung von Karzinomen kümmern, wenn nicht wir?"

Die Arbeitsmedizin und diejenigen, die von Prof. Manz' Forschungsarbeiten und klinischem Einsatz profitieren durften, werden ihm stets ein ehrendes Andenken bewahren.

Prof. Dr. med. Dennis Nowak München

\section{Korrespondenzadresse}

Prof. Dr. D. Nowak

Institut und Poliklinik für Arbeits-, Sozial- und Umweltmedizin, WHO Collaborating Centre for Occupational Health,

Klinikum der Universität München

Ziemssenstr. 1, 80336 München

Dennis.Nowak@med.uni-muenchen.de 


\section{Hier steht eine Anzeige.}

Springer 